\title{
The open healing approach and the use of platelet-rich growth factors for socket preservation
}

\author{
Alecsandru Ionescu ${ }^{1,2}$, Aliona Dodi' ${ }^{1}$, Vasileios Panagopoulos ${ }^{1}$, Laura Carstocea ${ }^{3}$, \\ Mihnea Ioan Nicolescu ${ }^{4,5}$, Augustin Mihai ${ }^{6}$, Gabriela Tanase ${ }^{6}$ \\ ${ }^{1}$ Aesthetics One Dental Center, Bucharest, Romania \\ ${ }^{2} \mathrm{PhD}$ Student, Department of Implantology, Faculty of Dentistry, \\ Carol Davila University of Medicine and Pharmacy, Bucharest, Romania \\ ${ }^{3} \mathrm{PhD}$ Student, Carol Davila University of Medicine and Pharmacy, Bucharest, Romania \\ ${ }^{4}$ Department of Histology, Faculty of Dentistry, Carol Davila University of Medicine and Pharmacy, \\ Bucharest, Romania \\ ${ }^{5}$ Victor Babes National Institute of Pathology, Bucharest, Romania \\ ${ }^{6}$ Faculty of Dentistry, Carol Davila University of Medicine and Pharmacy, Bucharest, Romania
}

\begin{abstract}
This clinical study evaluated the outcome of bone regeneration using an open-healing approach. We performed 80 atraumatic extraction with socket preservation in $n=69$ patients $(0.98$ sex ratio). Post extraction sockets were filled with platelet-rich autologous plasma factors Endoret ${ }^{\circledR}($ PRGF $®)$ clots covered with fibrin membrane and were left exposed during healing. The bone volume was measured and the need for an additional augmentation in the implant stage was assessed using CBCT scans at $\mathrm{t}=8$ weeks. This analysis showed that open healing technique using PRGF allowed uneventful healing and sufficient bone formation. Thereby, soft-tissue problems associated with extensive flap mobilization and tension may be avoided. We concluded that the use of PRGF in socket preservation with the open healing approach can be favorable from both clinical and radiological points of view. Furthermore, there was almost no need for additional augmentation in the implant stage and the number of complications was limited.
\end{abstract}

Keywords: socket preservation, open healing, PRGF, flapless

\section{INTRODUCTION}

Oral implantology is evolving rapidly and it has been doing so since its inception. The latest developments are a direct result of patients' growing requirements for aesthetics and they range from new surgical techniques to innovative prosthetic systems and accessories. In order to obtain a stabile functional and aesthetic result, the augmentation of hard and soft tissues became a standard procedure for many oral surgeons (1).

Currently, tooth loss is often accompanied either by immediate implant placement or by a socket preservation procedure. Recent studies have demonstrated that immediate implantation will not prevent resorption of the alveolar ridge, as previously thought (2). However, the use of immediate implantation techniques without raising a muco-periosteal flap, combined with a bone graft in the gap left between the implant and the post extraction socket walls, led to osseointegration accompanied by high stability of bone and the resulted soft tissue $(3,4,5)$. The alveolar preservation procedures are aimed at conserving (to a large extent) the horizontal and vertical dimensions of the crest, facilitating a straight forward insertion of the implant, preferably without additional grafting. 
The autologous bone harvested either intra- or extra- orally has been considered for many decades as the golden standard for augmentation procedures and some still describe it as such. However, over time, the reported failures and the morbidity of the donor site, especially in the case of bone harvesting from the iliac crest (as the pelvic and maxillary bones have different embryological origins and consequently a different metabolism) spurred the evolution of new techniques (6). Nowadays, guided bone regeneration (GBR) is the preferred technique among augmentation procedures for treatment of alveolar ridge defects. It mainly refers to the use of barrier membranes that will separate and protect the grafting material furthermore covered by the flap. The open healing approach is an alternative for ridge preservation where the resorbable membranes are left deliberately uncovered by the flap for a guided secondary healing $(7,8)$.

During the last years, the use of different types of growth factors in oral surgery and implantology significantly increased, due to clinical advantages and the potential to improve patient's post-operative quality of life (9). In this context, we have analyzed the particularities of the growth factors use with the open healing concept in socket preservation.

\section{MATERIALS AND METHODS}

This study was conducted between 2014 and 2018 in a private dental practice. The study protocol is in accord with the Helsinki Declaration of 1975, revised in 2000 and was approved by the Ethics Committee. Every person involved in study signed an informed consent. Patients with hopeless teeth with indication for extraction and implant therapy were recommended for the study. The sites with bone wall defects present after the extractions, the sites with height loss of more than $15 \%$ of the alveolar walls compared to the adjacent teeth or the sites where immediate implant placement was possible were excluded from this study. There were 69 patients (sex ratio 0.98 ), with 80 surgical sites that presented all alveolar walls after extraction and the indication for two stage approach, with implant placement in the second stage. After the hopeless teeth were extracted atraumatically, the extraction sockets were cleaned, and all granulation tissue was removed carefully. The surgical interventions were performed and the pre- and postoperative care was administered according to our standard procedures for the open healing approach. A platelet-rich autologous plasma factors blood clot was applied into the socket respecting the manufacturer's instructions and was covered with the fibrine membrane. No flap or deperiostation were performed. The fibrine membrane was stabilized with a PTFE continuous suture $\left(\right.$ Coreflon $^{\circledR}$, Implacore Sp. z o.o. Poznan, Poland) that was applied to the free gingival margins.

Based on the previous studies and the results obtained in the regeneration of hard and soft tissue with the conventional techniques, we chose Endoret $^{\circledR}\left(\mathrm{PRGF}^{\circledR}\right)$ (BTI Biotechnology Institute, Vitoria-Gasteiz, Spain) to be used in this study. This system is used in oral surgery to promote bone and adjacent soft tissue regeneration in post-extraction defects. The method consists of filling and sealing the post-extraction defects with the standard biological matrix and with autologous fibrin. These platelet-rich autologous plasma factors influence a series of biological processes that favor cellular migration, growth and morphogenesis.

The main criteria for selecting the system for socket preservation using the open healing protocol was that Endoret ${ }^{\circledR}\left(\mathrm{PRGF}^{\circledR}\right)$ accelerate the "on site" formation process of a biodegradable fibrin matrix that acts as a temporary "scaffold" that facilitates tissue regeneration. Also, unlike other systems available, the leukocyte-free formula diminishes their proinflammatory action. The controlled activation, together with the formation of the matrix and the gradual release of growth factors make the method more effective over time.

Application of Endoret ${ }^{\circledR}\left(\mathrm{PRGF}^{\circledR}\right)$ in the post-extraction socket is performed according to the manufacturer's indications. (10). Conventional protocol of blood extraction and centrifugation is followed. The blood is extracted directly to the Endoret $^{\circledR}$ extraction tubes that are supplied in the KMU15 KIT using a wing set and an adapted hood so that the collection is performed in a closed circuit. The extraction tubes are processed using the Endoret $^{\circledR}\left(\mathrm{PRGF}^{\circledR}\right)$ centrifugation technique with the PRGF System IV centrifuge and following the protocol established for this purpose. For use in the post-extraction socket both fractions obtained 
(fraction 1 and 2) are activated in the fractioning tubes (white cap) supplied in the KMU15 KIT. Then, the activated fractions are placed in a glass container and kept at $37^{\circ} \mathrm{C}$ until the separation of membrane (fraction 1) and the clot (fraction 2) that will be inserted into the alveolus. (Fig. 1a, 1b). The membrane obtained from fraction 1 was prepared in the special conformer container at $0,5 \mathrm{~mm}$ thickness (Fig. 1c).

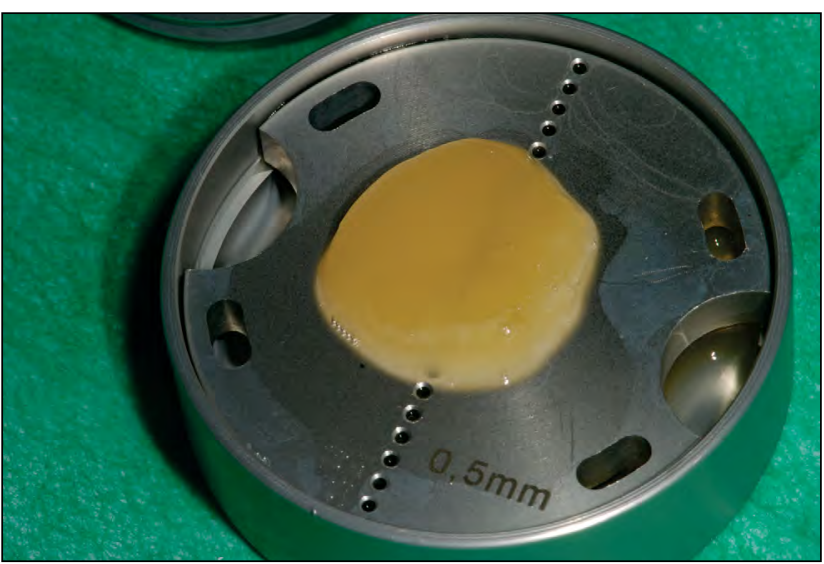

FIGURE 1a. Fibrine membrane shaper

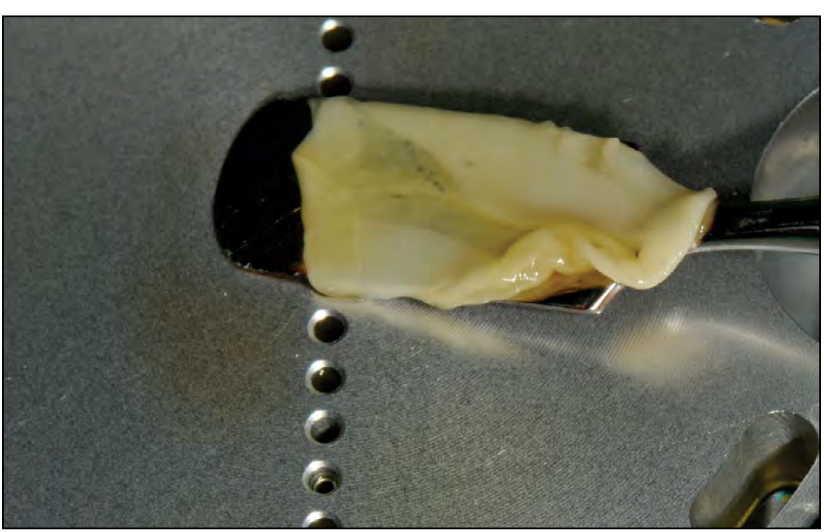

FIGURE 1b. PRGF fibrine membrane

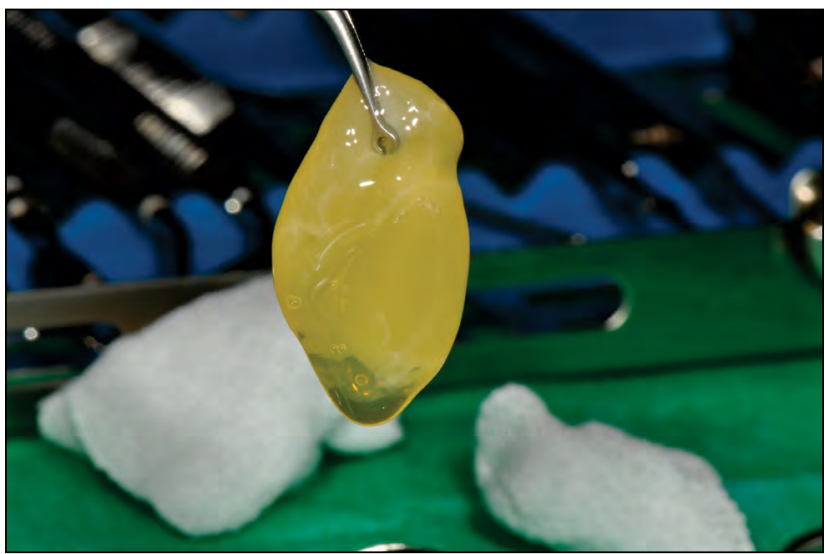

FIGURE 1c. PRGF clot

Antibiotics were not prescribed. Indications for cleaning and special care of the clinical sites were given. Painkillers and non-steroidal anti-inflammatories were recommended just when needed. Suture removal took place after two weeks. In order to allow maturation of bone and soft tissue, sites were allowed to heal for at least ten weeks before implant placement or secondary augmentation procedures were planned and performed. An example of a clinical case is shown in Figures 2a-m.

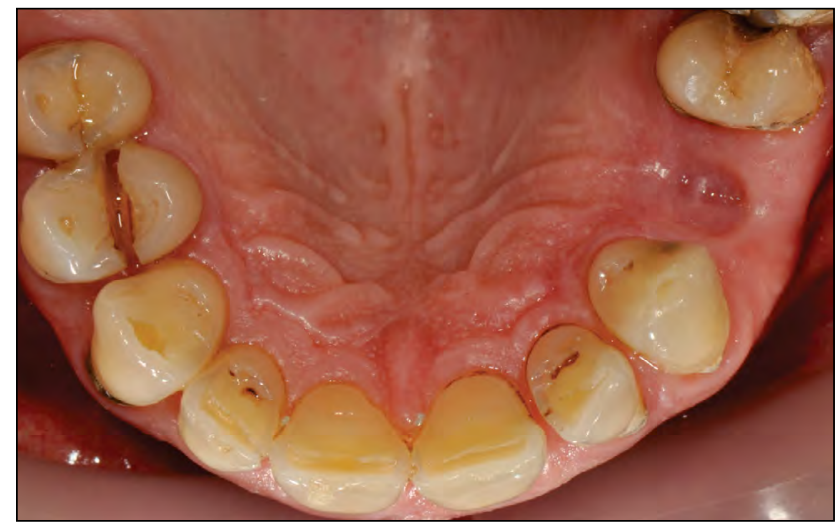

FIGURE 2a. Initial clinical situation; \#14 root fracture

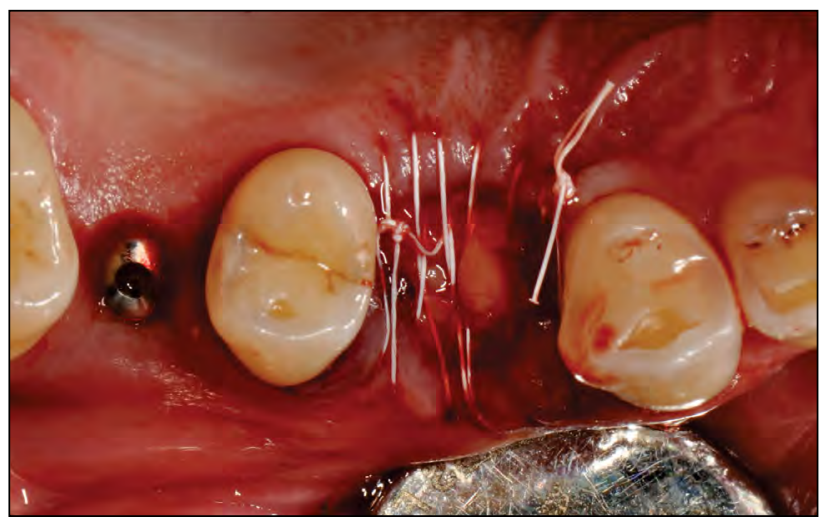

FIGURE 2b. Alveola of \#14 fulfilled with PRGF clot that was covered by PRGF fibrine membrane and fixed with PTFE continuous suture with no tension; no deperiostation was performed

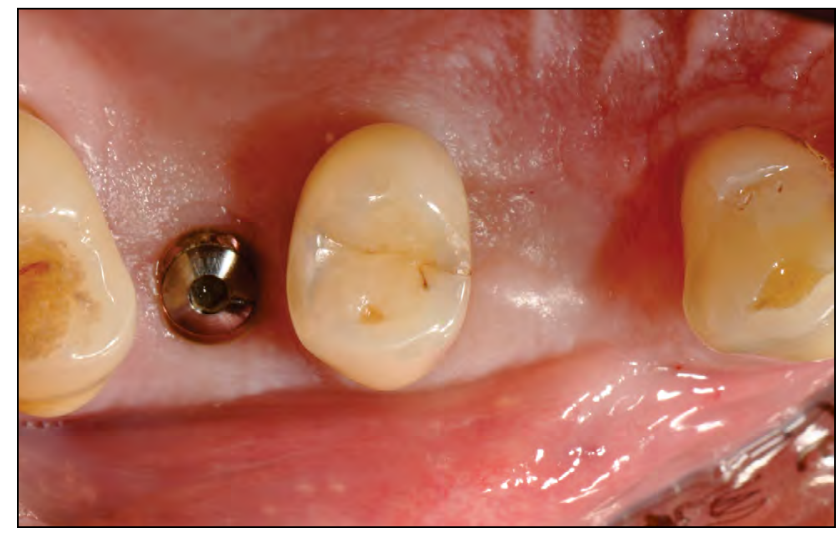

FIGURE 2c. Healed ridge prior to implant placement 


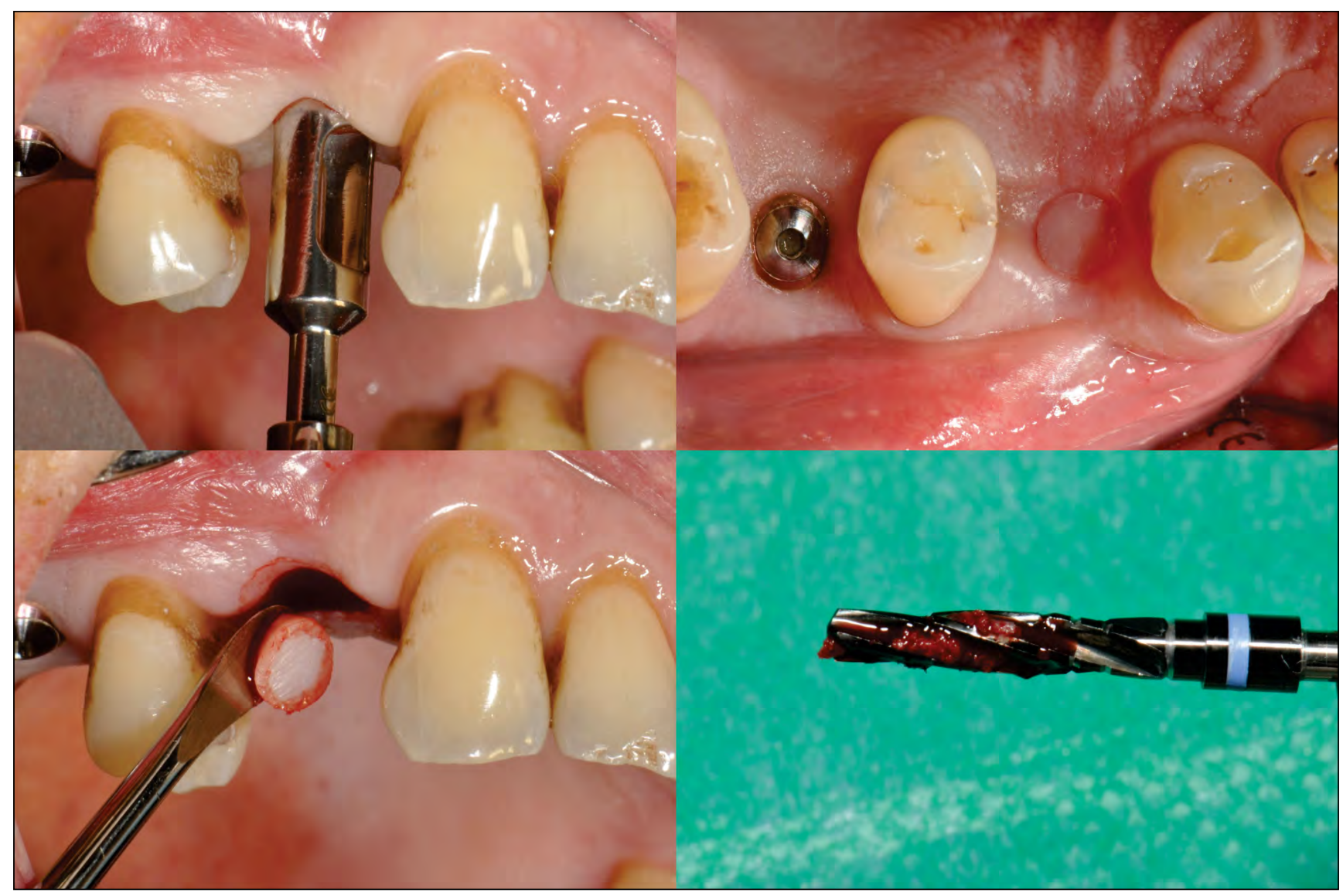

FIGURE 2d. Tissue punch, biological drilling, bone harvest

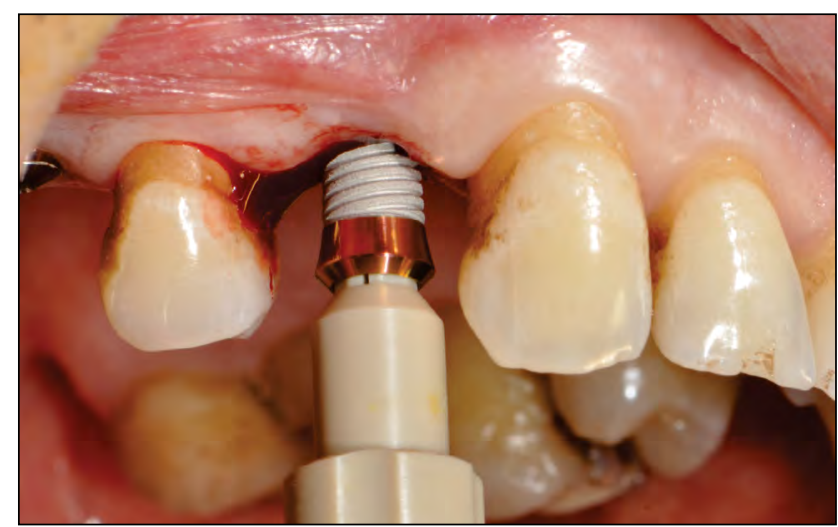

FIGURE 2e. Flapless implant insertion

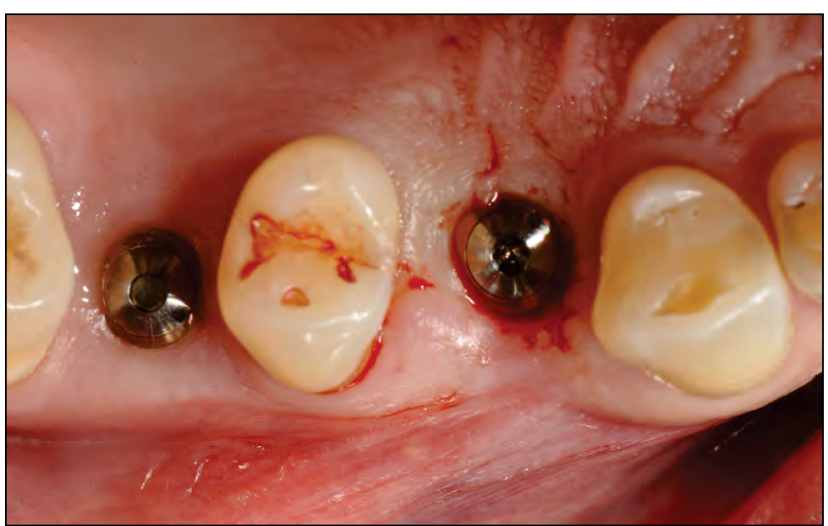

FIGURE 2f. Implant in situ
In some cases, multiple neighboring sockets were treated. These sites were defined as a single surgical area.

The aim of the study was to analyze whether using PRGF in socket preservation is suitable with the open healing technique in order to generate sufficient bone volume during healing and also considerably reduce the healing period to only $8-10$ weeks compared with deproteinized bovine bone and collagen membranes alternative. The primary outcome parameter was the bone volume obtained after ten weeks, measured on CBCT scans. The secondary outcome parameter was the need to perform an additional augmentation prior to or during the implant procedure. The treatment was judged to be successful if flapless implant placement was possible with no additional healing time and no additional augmentation were needed. 


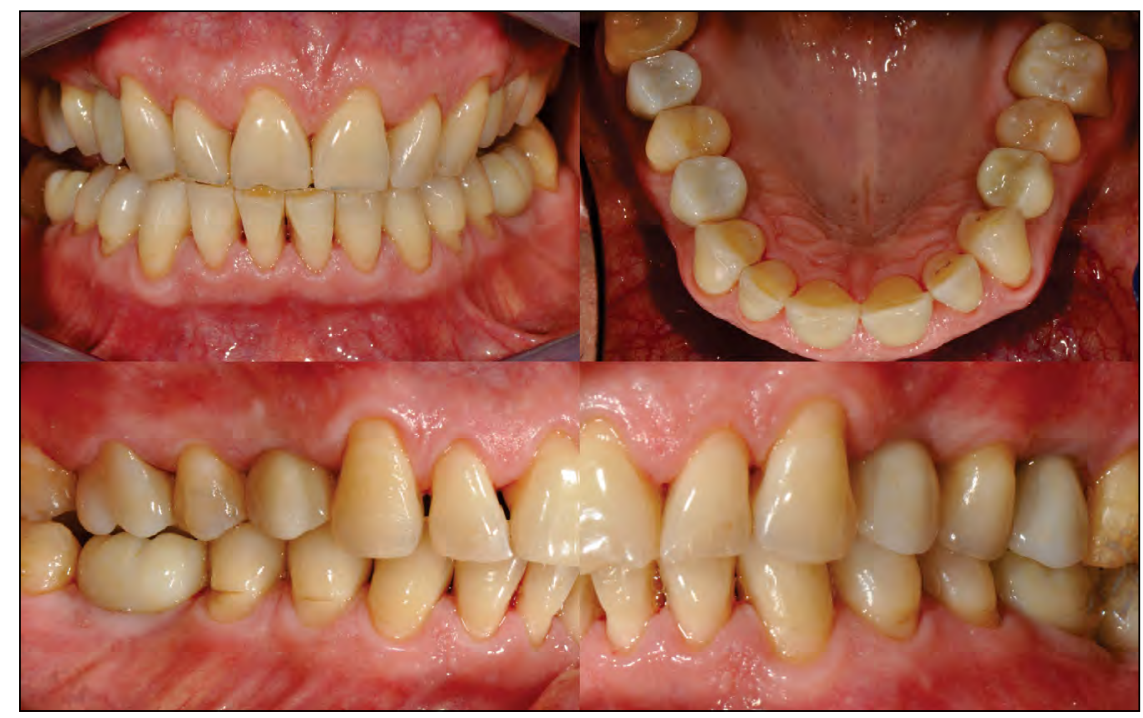

FIGURE 2g. Prosthetic restoration

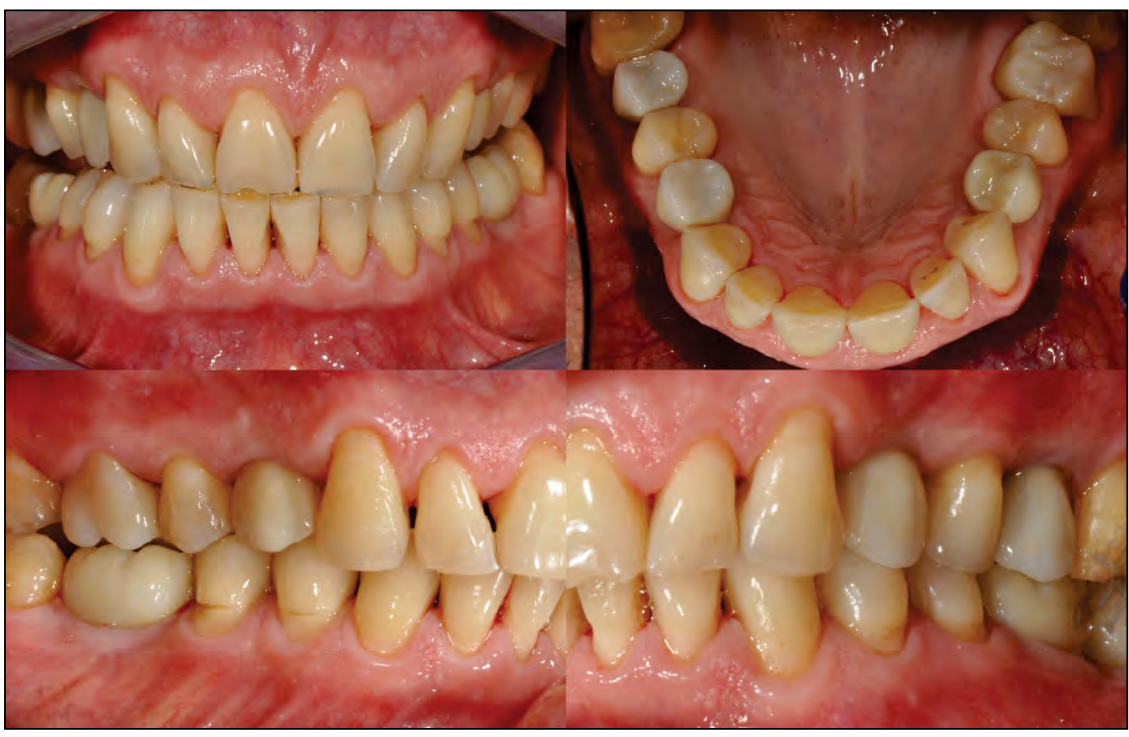

FIGURE 2h. Clinical follow up, 3 years

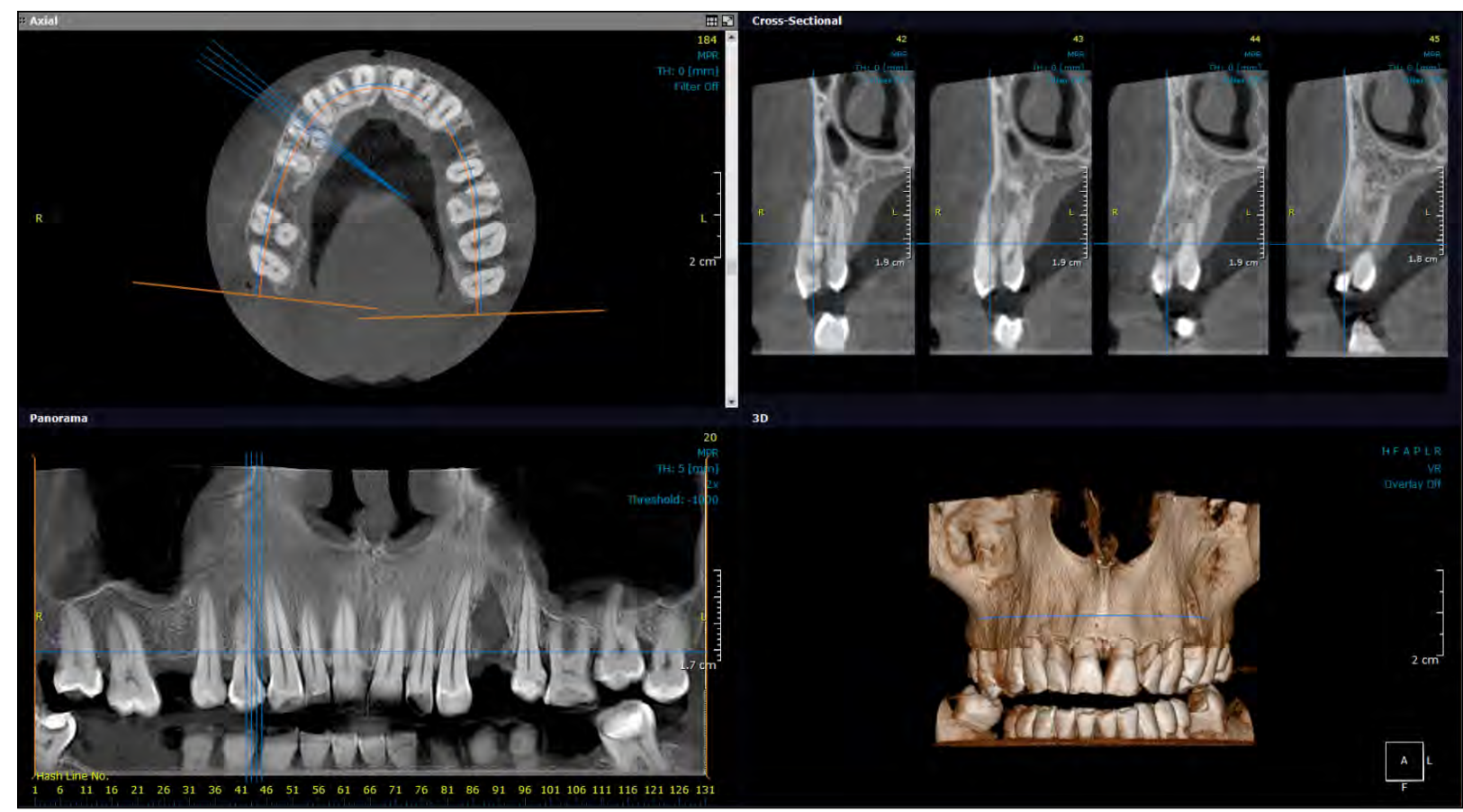

FIGURE 2i. CBCT scan before extraction of \#14 


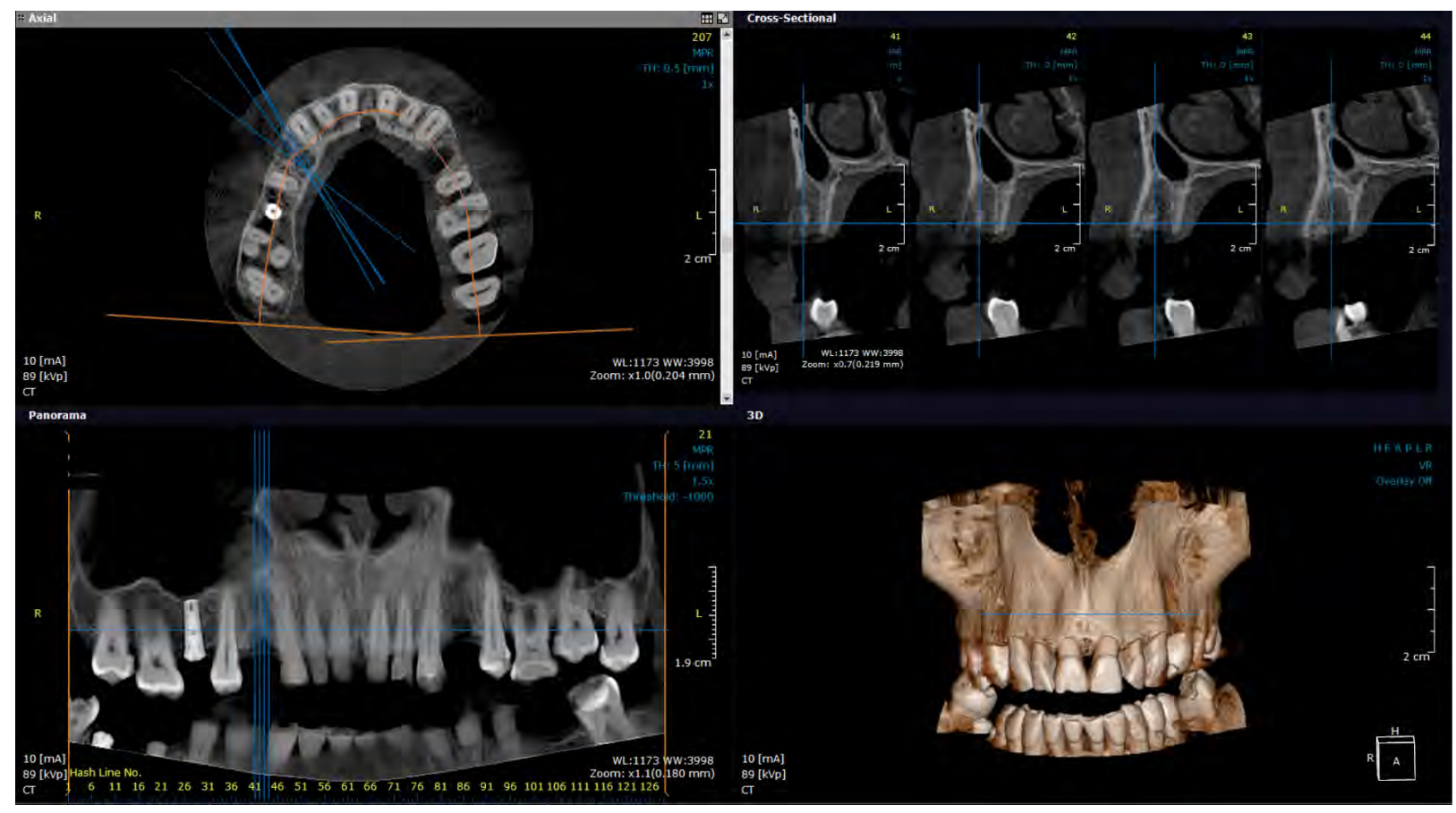

FIGURE 2j. CBCT scan 8 weeks after extraction of \#14 using the open healing technique and PRGF

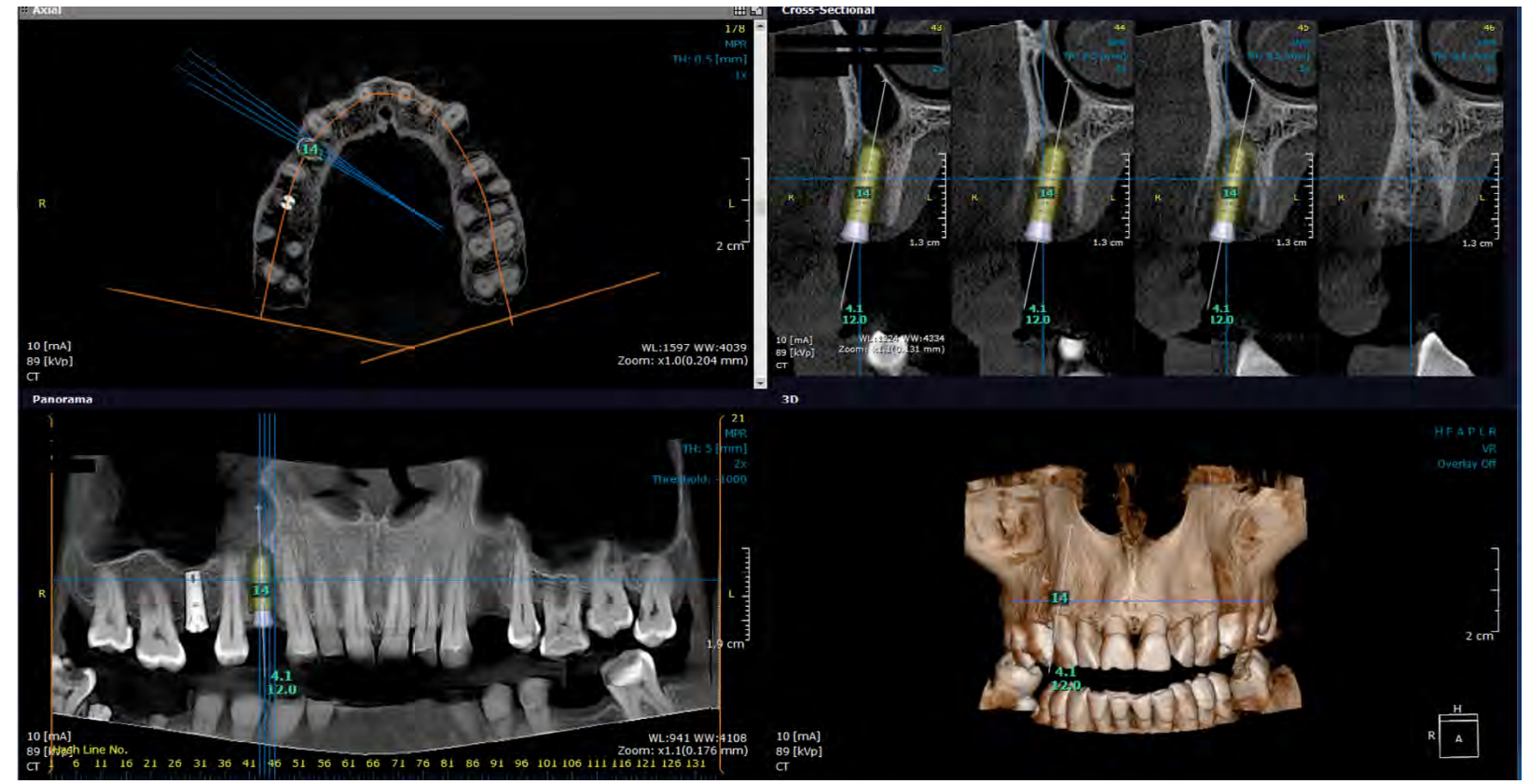

FIGURE 2k. Implant 3D simulation 


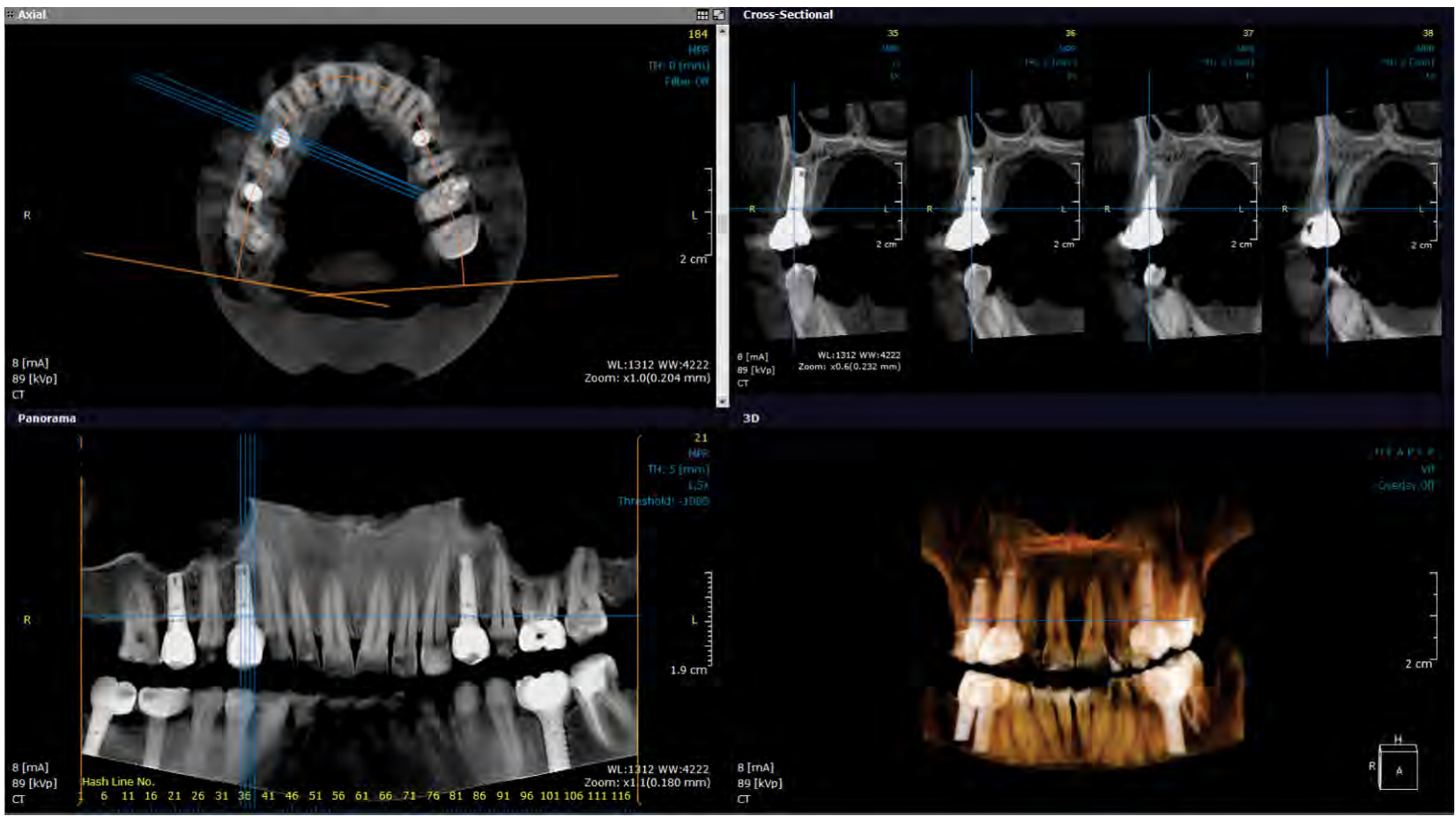

FIGURE 2I. CBCT scan 3 years follow up

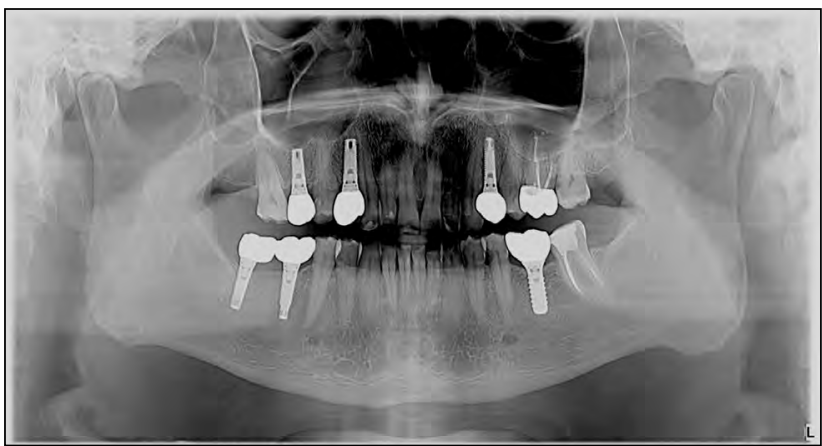

FIGURE 2m. Panoramic $x$-ray 4 years

TABLE 1. Number of additional augmantations needed prior to implant placement

\begin{tabular}{|l|c|}
\hline $\begin{array}{l}\text { Additional augmentation } \\
\text { prior to implant placement }\end{array}$ & $\begin{array}{c}\text { Number of augmented sites } \\
\text { (\%) }\end{array}$ \\
\hline Not necessary & $78(97,5 \%)$ \\
\hline Planned & $1(1,25 \%)$ \\
\hline Unplanned & $1(1,25 \%)$ \\
\hline
\end{tabular}

The CBCT scans were performed with the same investigation unit Cranex 3D (Soredex, Helsinki, Finland/KaVo Dental Gmbh, Biberach, Germany) and analyzed with the OnDemand 3D software (CyberMed, Yuseong-gu, Daejeon, Korea).

\section{RESULTS}

During the observation period, a total of 78 patients with 89 surgical areas were treated using PRGF with the open-healing approach. Nine patients did not make the appointment for implant placement, so they were excluded from the study. Therefore, the analysis included 80 surgical areas in 69 patients (49.6\% male and $50.4 \%$ female). Mean patient age was $54.3 \pm 13.0$ years (aged 2988 years).

In all cases, the extractions were performed atraumatically, sectioning the roots wherever needed, having as main purpose the maintenance of the surrounding bone walls and the inter-radicular septum. Also, periosteal incisions and flap mobilization were avoided.

The patients received a specific schedule for control and follow up appointments. Clinical observations were made at $24 \mathrm{~h}, 48 \mathrm{~h}, 7$ days and 14 days. The suture was removed after 14 days. Clinical outcome was observed, checking parameters such as inflammation, swelling, pain and soft tissue secondary healing. Patients received appointments for CBCT scan after eight to ten weeks in order to analyze the bone volume and the possibility of scheduling the implant placement stage. From the 80 sites, in 7 cases the CBCT scan showed areas of radio translucency, interpreted as immature bone formation. We assessed that these sites required additional healing time. In other 2 cases, the CBCT scans showed unfavorable outcomes and additional augmentation was needed during the implant placement procedures. There was no situation that needed additional augmentation in a separate stage that 
postponed the implant placement. For the other 71 sites, implant placement was performed flapless at week $10 \pm 2$. CBCT scan measurements showed that socket was regenerated $80 \%$ of baseline volume. Also, the width of the socket was $97.02 \pm 1 \%$ maintained compared with the baseline and the height was also maintained at $96 \pm 1,7 \%$

TABLE 2. Clinical outcome at 8 weeks, associated with the CBCT scan result

\begin{tabular}{|l|c|}
\hline Tissue healing & $\begin{array}{c}\text { Number of } \\
\text { augmented sites (\%) }\end{array}$ \\
\hline $\begin{array}{l}\text { Good clinical outcome + favorable } \\
\text { CBCT scan result }- \text { implant insertion } \\
\text { performed at } 10 \pm 2 \text { weeks }\end{array}$ & $66(82,5 \%)$ \\
\hline $\begin{array}{l}\text { Satisfactory soft tissue outcome }+ \\
\text { favorable CBCT scan result }- \text { implant } \\
\text { insertion performed at } 10 \pm 2 \text { weeks }\end{array}$ & $5(6,25 \%)$ \\
\hline $\begin{array}{l}\text { Satisfactory soft tissue outcome }+ \\
\text { unsatisfactory CBCT scan result }- \\
\text { implant postponed for } 4 \text { weeks, no } \\
\text { additional augmentations needed }\end{array}$ & $7(11,25 \%)$ \\
\hline $\begin{array}{l}\text { Unsatisfactory soft tissue outcome } \\
+ \text { CBCT scan result }- \text { additional } \\
\text { augmentations needed in implant } \\
\text { insertion surgical stage }\end{array}$ & $2(2,50 \%)$ \\
\hline
\end{tabular}

TABLE 3. Surgical technique used for implant placement after socket healing

\begin{tabular}{|l|c|}
\hline $\begin{array}{l}\text { Implant placement in the augmented } \\
\text { site }\end{array}$ & $\begin{array}{c}\text { Number of } \\
\text { augmented sites (\%) }\end{array}$ \\
\hline Flapless approach & $72(90 \%)$ \\
\hline Flap approach & $8(10 \%)$ \\
\hline
\end{tabular}

\section{DISCUSSIONS}

The standard procedure for tooth extraction generates alveolar bone loss due to bone remodeling. In the past, the main concern after the healing following a tooth extraction was the stability of the removable prostheses (11). Nowadays, implant therapy options have changed the treatment plan philosophies for replacing the hopeless teeth that are generating edentulous ridges. Even though, in order to facilitate the implant therapy, ridge resorption caused by tooth extraction should be limited or even eliminated. In this analysis, all tooth extractions were performed atraumatically. It was proven that atraumatic extraction is not sufficient for maintaining the bone volume. There are many socket preservation techniques as well as grafting materials that were analyzed in different studies that were published in the last decade $(12,13,14)$.

The positive result regarding the low necessity of an additional augmentation prior or during the implant phase indicates that open healing approach using PRGF may be a suitable clinical procedure. However, prospective studies should compare the outcome of open and closed healing under standardized clinical conditions.

\section{CONCLUSIONS}

The conclusions confirm the hypothesis that the use of PRGF in socket preservation with the open healing approach can be favorable from both clinical and radiological points of view. Furthermore, there was almost no need for additional augmentation in the implant stage and there were no complications reported.

This analysis indicates that open healing technique using PRGF allows uneventful healing and sufficient bone formation. Thereby, soft-tissue problems associated with extensive flap mobilization and tension may be avoided. Although the use of PRGF in open healing approach is limited to sites having all four surrounding bone walls, this can be exceeded by mixing the PRGF with deproteinized bovine bone in order to gain a volumetric stability. Prospective studies with control groups are needed to further investigate this surgical approach.

Furthermore, since the use of open healing yielded very good results in a single center patient group, we may presume that it could have the potential to become a wider spread clinical option.

\section{REFERENCES}

1. Gaviria L, Salcido JP, Guda T and Ong JL, 2014. Current trends in dental implants. Journal of the Korean Association of Oral and Maxillofacial Surgeons, 40(2), pp.50-60.

2. Neves M, Correia A. and Alves CC, 2013. A novel approach to preserve the buccal wall in immediate implant cases: a clinical report. Journal of Oral Implantology, 39(2), pp.198-205.

3. Mehta H, Shah S, 2015. Management of Buccal Gap and Resorption of Buccal Plate in Immediate Implant Placement: A Clinical Case Report. Journal of international oral health: JIOH, 7(Suppl 1), p.72.

4. Gotta SL, Reynolds MA, Saito H. and Tarnow DP, 2015. Flapless postextraction socket implant placement, part 2: the effects of bone grafting and provisional restoration on peri-implant soft tissue height and thickness-a retrospective study. Int J Periodontics Restorative Dent, 35(6), pp.803-809

5. Albiero AM, Benato R and Degidi M, 2014. Buccal plate regeneration with immediate post extraction implant placement and restoration. Int J Periodontics Restorative Dent, 34(4), pp.e67-e72. 
6. Costa LM, Sauvigné T and Guiol J, 2016. Morbidity of autologous bone harvesting in implantology: literature review from 1990 to 2015. Revue de stomatologie, de chirurgie maxillo-faciale et de chirurgie orale, $117(6)$, pp.388-402.

7. Taffet G, 2016. Open-healing approach to avoid flap mobilization and subsequent morbidity. Jurnal of Oral Science and Rehabititation, 2(4), pp.16-23

8. Ionescu A, Panagopoulos V and Taffet G, 2015. Open healing protocol as alternative ridge augmentation procedure in implant patients. Clinical Oral Implants Research, 26, p.441.

9. Anitua $E$, Orive $G$, Andia I. Use of PRGF to accelerate bone and soft tissue regeneration in post extraction sites. Implant Dialogue. 3-14.

10. Anitua E, Murias-Freijo A, Alkhraisat MH, Orive G. Clinical, radiographical, and histological outcomes of plasma rich in growth factors in extraction socket: a randomized controlled clinical trial. Clin Oral Investig. 2014 Jul 8.
11. Schettler D, 1991. Long time results of the Sandwich-technique for mandibular alveolar ridge augmentation. Deutsche Stomatologie (Berlin, Germany: 1990), 41(10), pp.376-378.

12. Kay SA, Wisner-Lynch L, Marxer M and Lynch SE, 1997. Guided bone regeneration: integration of a resorbable membrane and a bone graft material. Practical periodontics and aesthetic dentistry: PPAD, 9(2), pp.185-94.

13. Wang HL, Kiyonobu K and Neiva RF, 2004. Socket augmentation: rationale and technique. Implant dentistry, 13(4), pp.286-296.

14. Kim DM, De Angelis N, Camelo M, Nevins ML, Schupbach P. and Nevins M., 2013. Ridge preservation with and without primary wound closure: a case series. International Journal of Periodontics \& Restorative Dentistry, 33(1). 